\title{
Efecto del flushing sobre el desempeño reproductivo en ovejas de pelo en un centro de apoyo a la investigación y docencia en México
}

\author{
Effect of flushing on reproductive performance in hair sheep in a research \\ and teaching support center in Mexico
}

\section{Efeito do rubor no desempenho reprodutivo em ovelhas de cabelo em um}

\section{centro de apoio à pesquisa e ensino no México}

\author{
Gonzales Garzón Andrea Carolina1, Góngora Orjuela Agustín² y \\ Sánchez Gallegos Jaime ${ }^{3}$ \\ ${ }^{1}$ Medico Veterinario y Zootecnista, Universidad de los Llanos \\ ${ }^{2}$ Medico Veterinario PhD, Docente Universidad de los Llanos \\ ${ }^{3}$ Medico Veterinario PhD, Docente Colegio de Postgraduados, México \\ andrea.gonzalez.garzon@unillanos.edu.co
}

Recibido 10 de febrero 2020, Aceptado 23 de octubre 2021

\section{RESUMEN}

La producción ovina es un renglón importante de la economía pecuaria en México, la cual puede ser mejorada con la implementación de estrategias nutricionales y biotecnológicas. Este estudio evaluó el efecto del flushing en ovejas con condición corporal media (CCM) o baja (CCB) sobre el desempeño reproductivo en un centro de apoyo a la investigación y docencia en México. Se seleccionó un grupo de 42 ovejas multíparas de razas Pelibuey, Dorper, Hampshire, East friesian y Damara asignadas a 4 tratamientos $\mathrm{T} 1$ : ( $\mathrm{N}=10 \mathrm{CCM}$ con flushing), $\mathrm{T} 2$ : ( $\mathrm{N}=9 \mathrm{CCM}$ sin flushing), T3: ( $N=12$ CCB con flushing), $T 4$ : ( $N=11$ CCB sin flushing). El flushing consistió en una dieta basada en sorgo (proteína cruda: 14.76), se realizaron pesajes (antes, durante y después del experimento). Se sincronizaron con un $\mathrm{CIDR}^{\circledR}$, con retiro al día 9 y detectado el estro y monta natural inmediata. Al día 7 se aplicó $1 \mathrm{~mL}$ de Lutalyse $^{\circledR}$ (7.5 mg PGF2 $\alpha$ ). Se evaluó la tasa de ovulación y gestación por ultrasonografía al día 45 y 75, igualmente se midió la prolificidad y fecundidad. Las medias de los pesajes fueron: antes de flushing T1: 49.08; T2: 49.15; T3: 54.46 y T4: 52.43, durante flushing T1: 48.12; T2: 47.08; T3: 55.06 y T4: 
50.56 y post flushing T1: 49.42; T2: 49.11; T3: 55.00 y T4: 53.63. Las medias totales para los 3 pesajes fueron $\mathrm{P} 1=51.28 ; \mathrm{P} 2=50.21 ; \mathrm{P} 3=51.79$. En cuanto a las variables reproductivas no se encontraron diferencias estadísticamente significativas por medio de una prueba de Z; sin embargo las proporciones de los datos obtenidos muestran diferencias entre tratamientos, como los son: en la presentación del estro de los T1, T3 y T4 (90, 100 y 90.4\% respectivamente) frente al T2 (55.5\%); en la gestación el T1 (60\%) frente a T2, T3 y T4 (44.4, 41.7 y 10\% respectivamente) y respecto a la prolificidad los T1, T3 y T4 tuvieron un mayor número de crías/oveja (0.80; 1.00 y 0.90 respectivamente) respecto al T2 (0.33). El uso del flushing en animales con CCB antes de la monta o durante la sincronización con métodos farmacológicos mejora la fertilidad; pero es importante seguir supliendo los requerimientos de proteína para mejorar la fecundidad, disminuir las muertes neonatales y mejorar el peso al nacimiento de los borregos.

Palabras clave: Fecundidad, suplementación, gestación, pesaje, prolificidad.

\begin{abstract}
Sheep production is an important change in the livestock economy in Mexico, the quality can be improved with the implementation of nutritional and biotechnological strategies. This study evaluated the effect of flushing in sheep with medium body condition (CCM) or Low (CCB) body condition on reproductive performance in a Research and Teaching Support Center in Mexico. A group of 42 multiparous sheep of Pelibuey, Dorper, Hampshire, East Friesian and Damara breeds assigned to 4 treatments $\mathrm{T1}$ : ( $\mathrm{N}=10 \mathrm{CCM}$ with flushing), T2: ( $\mathrm{N}=9 \mathrm{CCM}$ without flushing), T3: ( $\mathrm{N}$ $=12 \mathrm{CCB}$ with flushing), T4: ( $\mathrm{N}=11 \mathrm{CCB}$ without flushing). The washing consists of a diet based on sorghum (Crude Protein: 14.76), weights (before, during and after the experiment). It is synchronized with a $\operatorname{CIDR}^{\circledR}$, with withdrawal on day 9 and detected the estrus and immediate natural riding. On day $7,1 \mathrm{~mL}$ of Lutalyse ${ }^{\circledR}(7.5$ mg PGF2 $\alpha$ ) was applied. The rate of ovulation and pregnancy by ultrasonography at day 45 and 75 was evaluated, prolificity and fertility were also measured. The weighing averages were: before rinsing T1: 49.08; T2: 49.15; T3: 54.46 and T4: 52.43, during washing T1: 48.12; T2: 47.08; T3: 55.06 and T4: 50.56 and after
\end{abstract}


discharge T1: 49.42; T2: 49.11; T3: 55.00 and T4: 53.63 . The total averages for the 3 weighings were $\mathrm{P} 1=51.28 ; \mathrm{P} 2=50.21 ; \mathrm{P} 3=51.79$. Regarding reproductive variables, no statistically determined differences were found by means of a $Z$ test; however, the proportions of the data that change the differences between treatments, such as: in the presentation of the estrus of T1, T3 and T4 (90, 100 and $90.4 \%$ respectively) versus T2 (55.5\%); in pregnancy, T1 (60\%) versus T2, T3 and T4 (44.4, 41.7 and 10\% respectively) and regarding prolificacy, T1, T3 and T4 had a greater number of offspring/sheep $(0.80,1.00$ and 0.90 respectively) with respect to T2 (0.33). The use of rinsing in animals with CCB and CCM before the mountain or during synchronization with pharmacological methods improves fertility; but it is important to continue supplying protein requirements to improve fertility, reduce neonatal deaths and improve the weight at birth of sheep.

Keywords: Fertility, supplementation, pregnancy, prolificity, weighing.

\section{RESUMO}

A produção ovina é uma linha importante da economia pecuária no México, que pode ser melhorada com a implementação de estratégias nutricionais e biotecnológicas. Este estudo avaliou o efeito do rubor em ovelhas com condição corporal média (CCM) ou Baixa (CCB) sobre o desempenho reprodutivo em um Centro de Apoio ao Ensino e Pesquisa no México. Um grupo de 42 ovelhas multíparas das raças Pelibuey, Dorper, Hampshire, East Friesian e Damara atribuídos a 4 tratamentos $\mathrm{T} 1$ : $(\mathrm{N}=10 \mathrm{CCM}$ com flushing), T2: ( $\mathrm{N}=9 \mathrm{CCM}$ sem flushing), T3: ( $N=12$ CCB com descarga), T4: ( $N=11$ CCB sem descarga). A lavagem consistiu de dieta à base de sorgo (proteína bruta: 14,76), sendo realizadas pesagens (antes, durante e após o experimento). Foram sincronizados com $\mathrm{CIDR}^{\circledR}$, com retirada no $9^{\circ}$ dia e detecção do estro e montagem natural imediata. No dia 7 , foi aplicado $1 \mathrm{~mL}$ de Lutalyse ${ }^{\circledR}$ (7.5 mg PGF2 $\alpha$ ). A ovulação e a taxa de gestação foram avaliadas por ultrassonografia nos dias 45 e 75, e a prolificidade e a fertilidade também foram medidas. As médias das pesagens foram: antes da lavagem T1: 49.08; T2: 49.15; T3: 54.46 e T4: 52.43, na lavagem T1: 48.12; T2: 47.08; T3: 55.06 e T4: 50.56 e T1 pós-lavagem: 49.42; T2: 49.11; T3: 55.00 e T4: 53.63. As médias 
totais das 3 pesagens foram $\mathrm{P} 1=51.28 ; \mathrm{P} 2=50.21 ; \mathrm{P} 3=51.79$. Em relação às variáveis reprodutivas, não foram encontradas diferenças estatisticamente significativas por meio do teste Z; Porém, as proporções dos dados obtidos mostram diferenças entre os tratamentos, tais como: na apresentação do estro em T1, T3 e T4 (90, 100 e 90.4\% respectivamente) versus T2 (55.5\%); na gestação, T1 (60\%) em relação a T2, T3 e T4 (44.4, 41.7 e 10\% respectivamente) e quanto à prolificidade, T1, T3 e T4 tiveram maior número de filhos/ovelhas $(0.80,1.00$ e 0.90 respectivamente) com em relação a T2 (0.33). O uso de lavagem em animais com CBC antes do acasalamento ou durante a sincronização com métodos farmacológicos melhora a fertilidade; mas é importante continuar atendendo aos requisitos de proteína para melhorar a fertilidade, reduzir as mortes neonatais e melhorar o peso ao nascer das ovelhas.

Palavras-chave: Fertilidade, suplementação, gestação, pesagem, prolificidade.

\section{INTRODUCCIÓN}

Entre las especies menores, las ovejas de pelo han ido evolucionando bajo la influencia selectiva de la naturaleza y del hombre, progresivamente se han venido adaptando a los diferentes ambientes tropicales, resultando en la actualidad de gran importancia económica para los países en vías en desarrollo. De forma paralela, la nutrición representa un pilar importante para los sistemas productivos, ya que de ella depende la rentabilidad de la empresa ganadera. De las divisiones que tiene la zootecnia entre ellas la nutrición, reproducción, alimentación y mejoramiento genético; la primera de ellas, es la más importante desde el punto de vista cuantitativo y económico, ya que dependiendo de la especie animal representa entre el 60 y $85 \%$ de los costos de producción (Gozonga, 2016).

La ovinocultura mexicana está considerada como una de las mejores en el mundo, tal como fue catalogada durante la X Asamblea General Ordinaria de la Unión Nacional de Ovinocultores (ONU) de México. Culturalmente México es uno de los principales consumidores de carne ovina, por lo tanto, el gobierno mexicano se ha encargado en impulsar la producción y exportación ovina con el fin de no sólo 
satisfacer su necesidad, si no de que sea visto como uno de los países con mejores avances científicos, tecnológicos y genéticos en esta producción. A pesar de lo anterior, la producción ovina mexicana, mantiene altos niveles de ineficiencia, por lo que se viene intentando un mejoramiento de las condiciones nutricionales asociados con procesos biotecnológicos, buscando una mayor eficiencia de este sistema productivo (SAGARPA, 2020).

El problema más grande que tiene la humanidad para enfrentar las próximas décadas es el uso de alimentos de calidad frente a las demandas de una población humana en constante crecimiento. La necesidad de producir alimentos de altos valores nutricionales e inocuos, demanda al sector agropecuario acciones para incrementar la producción agrícola y pecuaria (Salazar, 2015). Actualmente, el sector pecuario atraviesa una compleja transformación, puesto que se han venido adecuando los patrones de distribución geográfica de la producción pecuaria. Así mismo, se debe dar un uso racional de los recursos naturales. Por lo cual, se debe producir un volumen suficiente de alimento, esto registra un cambio en las especies utilizadas, con un crecimiento acelerado de la producción de especies monogástricas y una desaceleración de la producción de rumiantes.

La producción de carne ovina es una necesidad prioritaria del gobierno mexicano, su alto consumo, ha hecho que se considere un producto básico para la alimentación, principalmente de la región central (SAGARPA, 2015). A pesar de que México es considerado un país con un buen desarrollo de la producción ovina; su producción es insuficiente para abastecer la demanda del mercado nacional, por lo que tiene que importar un 30\% de Estados Unidos, Australia, Nueva Zelanda, Chile y Uruguay (Gozonga, 2016). Es por esto que se debe mejorar la eficiencia productiva, ya sea a través de estrategias, nutricionales, reproductivas, biotecnológicas entre otras.

El flushing es una estrategia de alimentación en la cual se aumenta la cantidad y calidad del alimento durante un periodo corto de tiempo (máximo 15 días). Los objetivos son generar un balance energético positivo para favorecer el retorno al estro, incrementar la tasa de ovulación (+ 2-3 óvulos) y mejorar el tamaño de las 
crías. La estrategia del flushing es modular nutricionalmente el periodo reproductivo a corto plazo, induciendo cambios en el estado metabólico del animal, pero sin causar cambios importantes en el peso o contenido de grasa corporal. Esta estrategia permite restablecer la ovulación en hembras que están en baja condición corporal; ya sea debido a bajos consumos durante o después del periodo de lactancia o debido a alimentación restringida lo que resulta en una menor liberación de LH (Martinez et al., 2015). De acuerdo a lo anterior, nace el cuestionamiento de este estudió, el cual fue evaluar el efecto del flushing sobre el desempeño reproductivo en ovejas de pelo.

\section{METODOLOGÍA}

Este estudio se llevó a cabo en las instalaciones del Colegio Posgraduados, Campus Montecillo, en las instalaciones del Laboratorio de Reproducción Ovina y Caprina (LaROCa). Ubicado en el kilómetro 36.5 de la Carretera Federal México Texcoco en Montecillo, Municipio de Texcoco, Estado de México $\left(19^{\circ} 29^{\prime} \mathrm{N}\right.$ y $98^{\circ}$ $53^{\prime} \mathrm{O}$ ), la temperatura oscila entre $6-16^{\circ} \mathrm{C}$ y las precipitaciones entre $500-1200 \mathrm{~mm}$.

Se utilizaron Inicialmente 52 hembras ovinas multíparas de razas: Pelibuey, Dorper, Hampshire, Damara y Eastfriesian. De este grupo se seleccionaron 42 las cuales fueron asignadas a un $\mathrm{G} 1 \mathrm{n}=19$ ovejas con un tiempo de post destete aproximado de 15 días, condición corporal 2 de 5 de acuerdo a la tabla propuesta por (Felice, 2013) clasificadas como condición corporal baja (CCB), con un peso promedio de $46 \mathrm{~kg}$. El segundo grupo G2 n=33 ovejas con 60 días post destete, condición corporal entre 3 sobre 5 clasificadas como condición corporal media (CCM) y un peso promedio de $54 \mathrm{Kg}$.

Los grupos G1 y G2 fueron a su vez subdivididos al azar en 2 grupos cada uno; por lo tanto, en total se manejaron 4 grupos tratamientos a evaluar. Los tratamientos quedaron conformados y nombrados de la siguiente forma:

TTO 1*: CCB con flushing ( $\mathrm{n}=10$ ovejas); TTO 2: CCB sin flushing ( $\mathrm{n}=9$ ovejas);

TTO 3*: CCM con flushing ( $n=12$ ovejas) y TTO 4: CCM sin flushing ( $n=11$ ovejas). 
Con el fin de evaluar en cada tratamiento la ganancia de peso, presentación del estro, tasa de concepción, tasa de fertilidad, tasa ovulatoria y prolificidad al implementar un flushing corto pre monta en ovejas de CCB y CCM (TTO ${ }^{*}$ y TTO $3^{*}$ ) comparándolo con los dos tratamientos restantes (TTO 2 y TTO 4), a base de una dieta normal.

La condición corporal fue evaluada de acuerdo con (Felice, 2013), donde se valoraron las características físicas de partes anatómicas como: las apófisis espinosas, el músculo del lomo y las apófisis transversas; también se tomó como referencia la marcación de las costillas, teniendo en cuenta que una marcación muy evidente, sería CCB y una marcación medianamente nula CCM. Cada tratamiento fue asignado en corrales separados y los animales identificados con collares de fleques de colores y un número de identificación en argollas metálicas con su respectiva cadena, para facilitar el manejo y distinción de cada tratamiento, con comederos de cemento y bebederos automáticos (Figura 1).
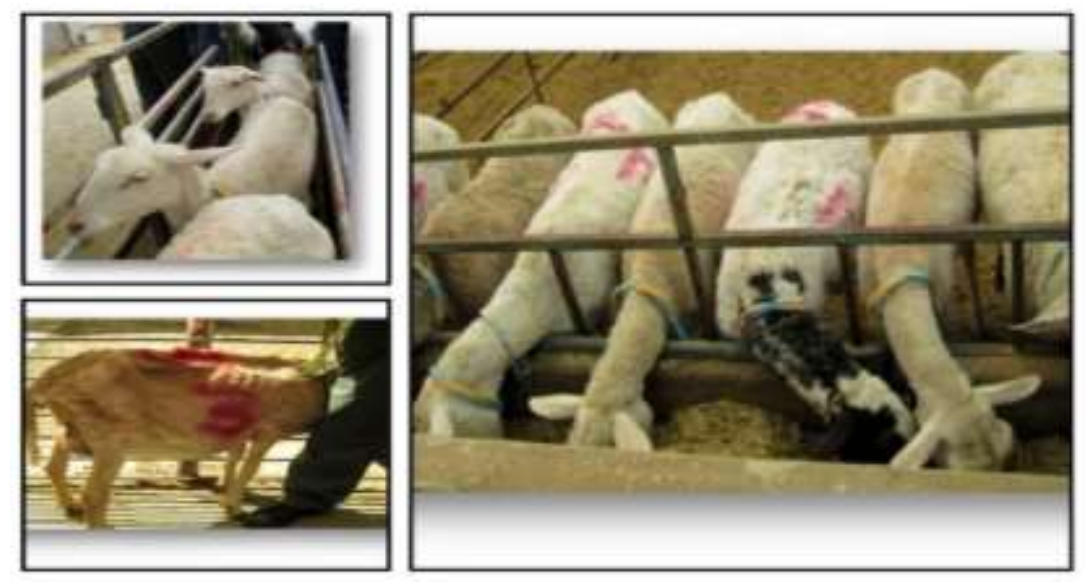

Figura 1. Grupo de ovejas multíparas con tiempo de post destete de 15 días aproximadamente (CCB). Fuente: Los autores.

Antes de iniciar el estudio se realizó desparasitación a todos los animales, se administró un $1 \mathrm{ml}$ de selenio y $2 \mathrm{ml}$ de vitamina B12 (Catosa ${ }^{\circledR}$ ) intramuscular (IM) dosis única. Además, se realizó el arreglo de pezuñas de todos los animales. (Martínez et al., 2018). 
La evaluación de la ganancia de peso se realizó mediante pesaje de los animales antes de iniciar el estudio, durante el programa de sincronización, cada tercer día hasta que se retiraron los dispositivos intravaginales y al finalizar el flushing en los tratamientos TTO 1 y TTO 3 . Una vez normalizada la dieta se realizaron pesajes cada 8 días de los animales. Para el pesaje se utilizó una manga de manejo para ovejas con una báscula electrónica.

Todas las ovejas del estudio fueron sometidas a un protocolo de sincronización del estro mediante dispositivos intravaginales $\mathrm{CIDR}^{\circledR}$ impregnados con $0.3 \mathrm{~g}$ de progesterona. Los dispositivos se colocaron por un periodo de nueve días, el séptimo día se administró $1 \mathrm{ml}$ de PGF2 $\alpha$ (Lutalyse ${ }^{\circledR}, 7.5 \mathrm{mg}$ ) IM a todos los tratamientos.

El Protocolo que se siguió para la colocación de dispositivos CIDR fue:

- Antes de proceder a la colocación de los dispositivos, se debe lubricar con antibiótico sin corticoides.

- Se introduce el dispositivo en el aplicador cuidando que la cuerda cuelgue hacia afuera.

- El aplicador se humedece igualmente.

- Para facilitar la colocación del dispositivo la hembra debe estar en posición de cuadripedestación. El aplicador con el dispositivo es introducido suavemente hasta el fondo de la vagina, teniendo en cuenta que las manijas del aplicador estén en posición horizontal (Figura 2).

- Una vez adentro el aplicador se debe girar hasta que las manijas del aplicador queden verticales (paralelas a los labios vulvares).

- Se libera el dispositivo y se retira el aplicador, halando la cuerda para cerciorarse de que el dispositivo no esté suelto. Para retirarlo se debe halar la cuerda suavemente con una leve inclinación hacia abajo, hasta que el dispositivo salga.

Cada cuatro horas después del retiro de los dispositivos intravaginales CIDR $^{\circledR}$, se inició observación del comportamiento del rebaño para detección del estro en todos 
los tratamientos durante 72 horas, seguido de detección cada 12 horas mañana y tarde hasta cumplir 120 horas post retiro CIDR $^{\circledR}$ con la ayuda de carneros con mandiles en cada corral durante media hora.
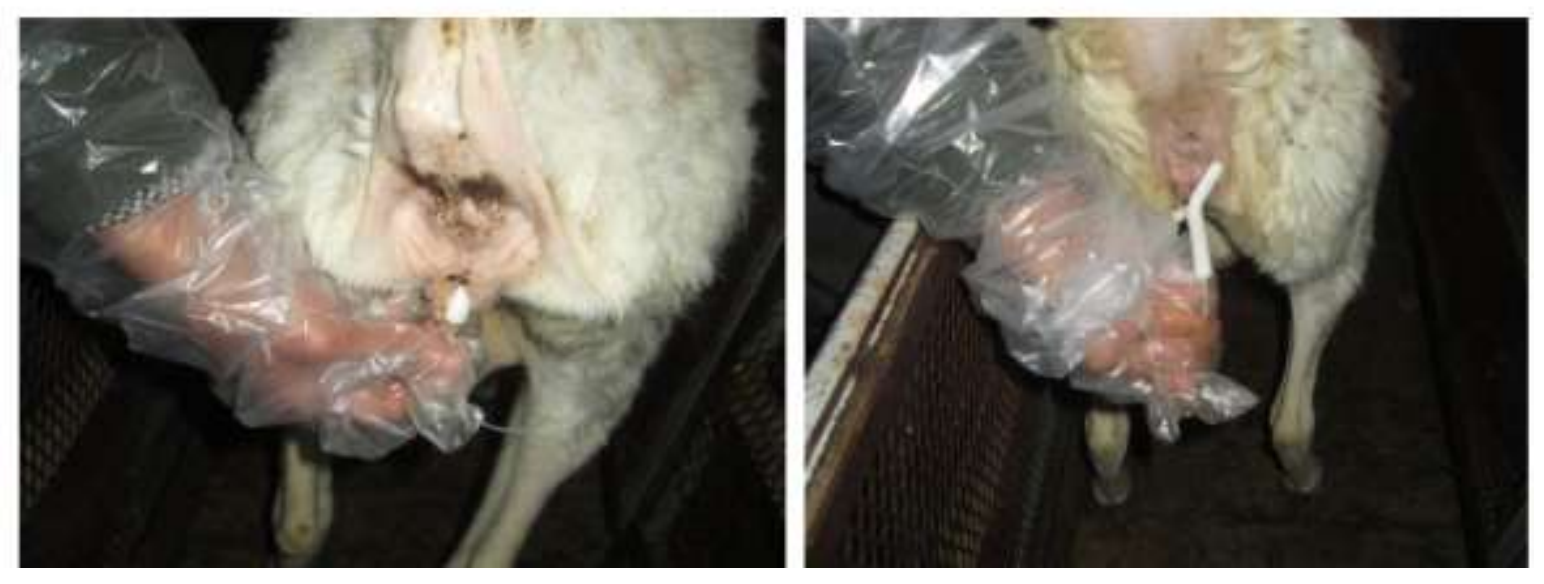

Figura 2. Colocación de dispositivos CIDR $^{\circledR}$. Fuente: Los autores.

Una vez el macho detectaba la hembra receptiva se retiraba del grupo y era identificada con un spray de color con números en orden dependiendo la hora de inicio del estro y permitida la monta natural (Figura 3).

Para la detección de los retornos al estro, el primero se vigiló a partir del día 13 hasta el día 22 post retiro CIDR $^{\circledR}$ (mañana y tarde). Para la detección del segundo retorno del estro se inició el día 28 hasta el día 34 post retiro CIDR $^{\circledR}$ una vez al día. Todas las hembras receptivas al primer y segundo retorno del estro fueron cubiertas mediante monta natural.

La tasa ovulatoria se evaluó en algunas hembras mediante ultrasonografía transrectal y realizado el conteo de cuerpos lúteos $(\mathrm{Cl})$ en cada ovario, en el décimo día post retiro del dispositivo CIDR $^{\circledR}$. Para este procedimiento los animales fueron sometidos a un ayuno de 12 horas, para evitar presencia de heces en intestino grueso y observar con mayor claridad los $\mathrm{Cl}$.

Para evaluar la variable de fertilidad se tuvo en cuenta el diagnóstico de gestación por ultrasonografía trans abdominal, a los 45 y 75 días del servicio. Para este procedimiento los animales se dejaron en ayuno durante 12 horas. 

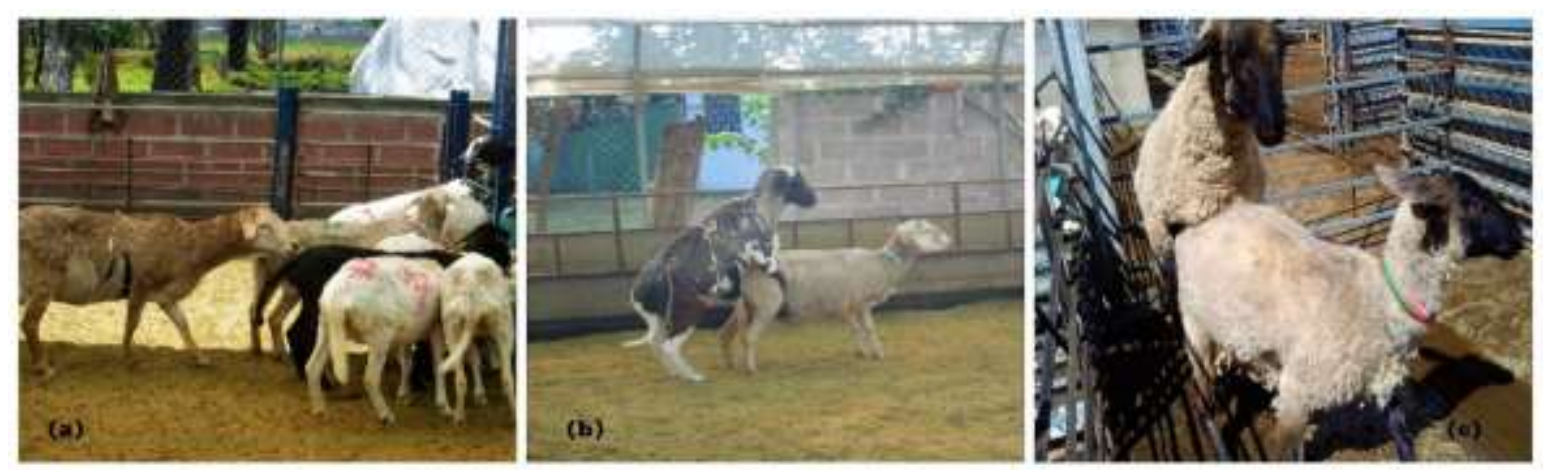

Figura 3. Respuesta a la sincronización en todos los grupos simultáneamente (a) detección de ovejas en celo (b) oveja receptiva (en celo) (c) monta natural oveja receptiva. Fuente: Los autores.

Para la medición de la tasa de concepción (TC), se tuvo en cuenta la siguiente fórmula:

$$
\% T C=\frac{\text { Núm.ovejas detectadas gestantes }(45 \text { días })}{\text { Núm.ovejas expuestas }} \times 100
$$

Esta tasa (TC) se relacionó directamente con la Tasa de fertilidad (TF), la cual se determinó a partir de esta fórmula:

$$
\% T F=\frac{N u ́ m . \text { ovejas paridas }}{N u ́ m . \text { ovejas expuestas }} \times 100
$$

\section{Alimentación flushing}

Al inicio del estudio todos los animales fueron alimentados con $1 \mathrm{~kg}$ de heno/animal/día más forraje verde picado. Cuando se inició el programa de sincronización del estro a las ovejas del TTO 1 y TTO 3 (CCB, CCM con flushing) se les cambio la dieta y se suministró la suplementación mediante la técnica flushing (Tabla 3) a una ración de $2 \mathrm{~kg}$ de dieta de Flushing/animal/día. Los tratamientos TTO 2 y TTO 4 (CCB, CCM sin Flushing) continuaron con la dieta inicial; Sin embargo, durante los nueve días del programa de sincronización del estro se les aumentó la ración a $2 \mathrm{~kg}$ de heno/animal/día. Al retirar los CIDR $^{\circledR}$ en el noveno día, 
volvieron a consumir $1 \mathrm{~kg}$ de heno/animal/día más forraje verde. En la Tabla 1 se presenta la composición de la dieta de las ovejas para los TTO 1 y 3.

Tabla 1. Flushing para Tratamientos TTO 1 y TTO 3.

\begin{tabular}{cc}
\hline Componentes & Valor \\
\hline Paja de avena $(\mathrm{Kg})$ & 3 \\
Carbonato calcio $(\mathrm{Kg})$ & 1 \\
Gluten de maíz $(\mathrm{Kg})$ & 4 \\
Melaza $(\mathrm{Kg})$ & 8.5 \\
Heno de avena $(\mathrm{Kg})$ & 3.5 \\
Pasta de canola $(\mathrm{Kg})$ & 9 \\
Sorgo $(\mathrm{Kg})$ & 47 \\
Cascarilla de soya $(\mathrm{Kg})$ & 13 \\
Urea $(\mathrm{Kg})$ & 0.5 \\
Salvado de trigo $(\mathrm{Kg})$ & 9.5 \\
Minerales $(\mathrm{Kg})$ & 1 \\
Total $(\mathrm{Kg})$ & 100 \\
Proteína cruda $(\%)$ & 14.76 \\
Energía metabolizable (Mcal/kg) & 2.71 \\
Fibra Cruda $(\%)$ & 11.27 \\
\hline
\end{tabular}

Fuente: Los autores

\section{Análisis estadístico}

Los datos fueron analizados por medio del programa estadístico SPSS realizándose una estadística descriptiva debido al tamaño de la muestra de cada grupo. Los pesajes se analizaron comparándolos entre tratamientos teniendo en cuenta el valor de la media poblacional de cada grupo. Las variables reproductivas como presentación del estro y repetición del estro se analizaron a través de proporciones a partir de los datos obtenidos.

\section{RESULTADOS Y DISCUSIÓN}

Los resultados obtenidos en el TTO $1^{*}$ Ovejas de condición corporal baja (CCB) con flushing y TTO $3^{*}$ Ovejas de condición corporal media (CCM) con flushing, no 
mostraron una diferencia significativa entre el pesaje 3 (después del flushing), manteniendo en promedio una diferencia de $1 \mathrm{~kg}$ aproximadamente entre pesajes. En el TTO 2 Ovejas de CCB sin flushing y el TTO 4 Ovejas de CCM sin flushing se evidenció una pérdida de peso en el pesaje 2 (final del flushing); pero para el pesaje 3 se evidenció nuevamente la recuperación de los kilogramos perdidos. Se puede concluir que el flushing en esta investigación no influyó positivamente sobre la ganancia de peso, quizás por tratarse de un flushing corto.

En la Tabla 2, se relaciona e integran los 4 tratamientos con los resultados de los pesajes 1, 2 y 3 ; es decir sólo se tuvo en cuenta los datos obtenidos en cada pesaje sin importar a qué tratamiento pertenecían. Esto se hizo con el fin de estimar qué tan significativo fue el aumento de peso en los animales sin importar la calidad del alimento. Se encontró una diferencia estadística significativa $(P<0.05)$ entre el pesaje 1 (antes del flushing) y el pesaje 3 (después del flushing). Se pudo evidenciar que a pesar de que no se obtuvo una ganancia de peso alta, se presentaron cambios en el peso de los animales, los cuales probablemente estuvieron relacionados con el cambio de la dieta y la cantidad de alimento suministrado.

Tabla 2. Comparación entre cada uno de los pesajes.

\begin{tabular}{|c|c|c|c|c|c|c|}
\hline \multirow{2}{*}{$\begin{array}{c}\text { (I) } \\
\text { Pesaje }\end{array}$} & \multirow{2}{*}{$\begin{array}{c}(\mathrm{J}) \\
\text { Pesaje }\end{array}$} & \multirow{2}{*}{$\begin{array}{l}\text { Diferencia } \\
\text { de medias } \\
\quad(I-J)\end{array}$} & \multirow{2}{*}{$\begin{array}{l}\text { Error } \\
\text { estándar }\end{array}$} & \multirow{2}{*}{ Sig. ${ }^{b}$} & \multicolumn{2}{|c|}{$\begin{array}{c}95 \% \text { de intervalo de } \\
\text { confianza para } \\
\text { diferencia }{ }^{b}\end{array}$} \\
\hline & & & & & $\begin{array}{l}\text { Límite } \\
\text { inferior }\end{array}$ & $\begin{array}{l}\text { Límite } \\
\text { superior }\end{array}$ \\
\hline \multirow{2}{*}{1} & 2 & $1.075^{*}$ & 0.219 & 0.000 & 0.526 & 1.624 \\
\hline & 3 & -0.507 & 0.317 & 0.354 & -1.302 & 0.287 \\
\hline \multirow{2}{*}{2} & 1 & $-1.075^{*}$ & 0.219 & 0.000 & -1.624 & -0.526 \\
\hline & 3 & $-1.582^{*}$ & 0.258 & 0.000 & -2.227 & -0.937 \\
\hline \multirow{2}{*}{3} & 1 & 0.507 & 0.317 & 0.354 & -0.287 & 1.302 \\
\hline & 2 & $1.582^{*}$ & 0.258 & 0.000 & 0.937 & 2.227 \\
\hline
\end{tabular}

Se basa en medias marginales estimadas

* La diferencia de medias es significativa en el nivel 0.05.

b Ajuste para varias comparaciones: Bonferroni.

Fuente: Los autores 
Banchero y Quintans, (2008) evaluaron diferentes tipos de flushing en ovejas con el fin de demostrar si el tipo de dieta mejoraba los parámetros reproductivos, especialmente prolificidad; a partir de esto surge la hipótesis de que la ganancia de peso sea dependiente del tipo de flushing (alimento) a pesar de ser corto, aunque el uso de flushing corto no busca aumentar significativamente la ganancia de peso en ovejas (Banchero et al., 2013).

Por lo tanto, el flushing realizado no influyó en la ganancia de peso en ninguno de los tratamientos; sin embargo, la condición corporal de las ovejas en los tratamientos 1 y 3 (con flushing) mejoró respecto a la presentada antes de iniciar el flushing. Resultados similares fueron obtenidos en Argentina, en un estudio donde suplementaron a un grupo con pellet de girasol frente al control, antes de la época de monta; encontrando un ligero aumento en el peso y condición corporal en las ovejas del grupo que fue suplementado respecto a las del grupo no tratado. Aunque estadísticamente no fue significativa está variación (Aguilar et al., 2009).

No obstante que con el flushing utilizado en este estudio no se obtuvo aumento de peso en las ovejas, algunos autores reportan que puede influir significativamente en el peso al nacimiento y crecimiento de sus borregos, como lo demostró De Azambuja et al., (2015) concluyendo además que el flushing en ovejas es realmente una práctica económicamente rentable. Otros estudios relacionan que un incremento en el peso mayor al $6 \%$ implementando la técnica flushing aumenta la tasa mellicera, es decir a mayor ganancia de peso mayor prolificidad (Álvarez et al., 2014).

La Gráfica 1, refiere las proporciones obtenidas del análisis de las variables reproductivas, presentación del estro, repetición del estro, gestación y parto. Debido al tamaño de la muestra no se encontraron diferencias significativas, pero al realizar análisis por medio de proporciones sí se evidencian. En cuanto a la presentación del estro el TTO $3^{*}$ tuvo mayor respuesta con un 100\%, seguido del TTO 4 (90.9\%) y el TTO $1^{*}$ (90\%), sin embargo, el TTO 2 tan solo obtuvo un $55.5 \%$ de todas las hembras evaluadas. En todos los tratamientos observados se logró una 
presentación de estro significativa de acuerdo con el programa de sincronización diseñado.

Al comparar los tratamientos de CCB con flushing y sin flushing, el TTO1* $(90 \%)$ y el TTO 2 (55.5\%) se encuentra una diferencia del 35\%, donde las ovejas de CCB evidenciaron mayor respuesta al programa de sincronización cuando se empleó la técnica flushing. Sin embargo, los tratamientos de CCM con flushing y sin flushing TTO $3^{*}(100 \%)$ y TTO 4 (90.9\%), con tan solo una diferencia del $9.1 \%$ permite corroborar que una condición corporal media favorece la respuesta positiva a protocolos de sincronización. Es por esto que al seleccionar hembras para la reproducción la condición corporal ideal es de 3 a 4/5 para iniciar programas de sincronización y obtener resultados que favorezcan la presentación del estro (Gottardi et al., 2014; Martínez et al., 2018; Sanchez et al., 1990).

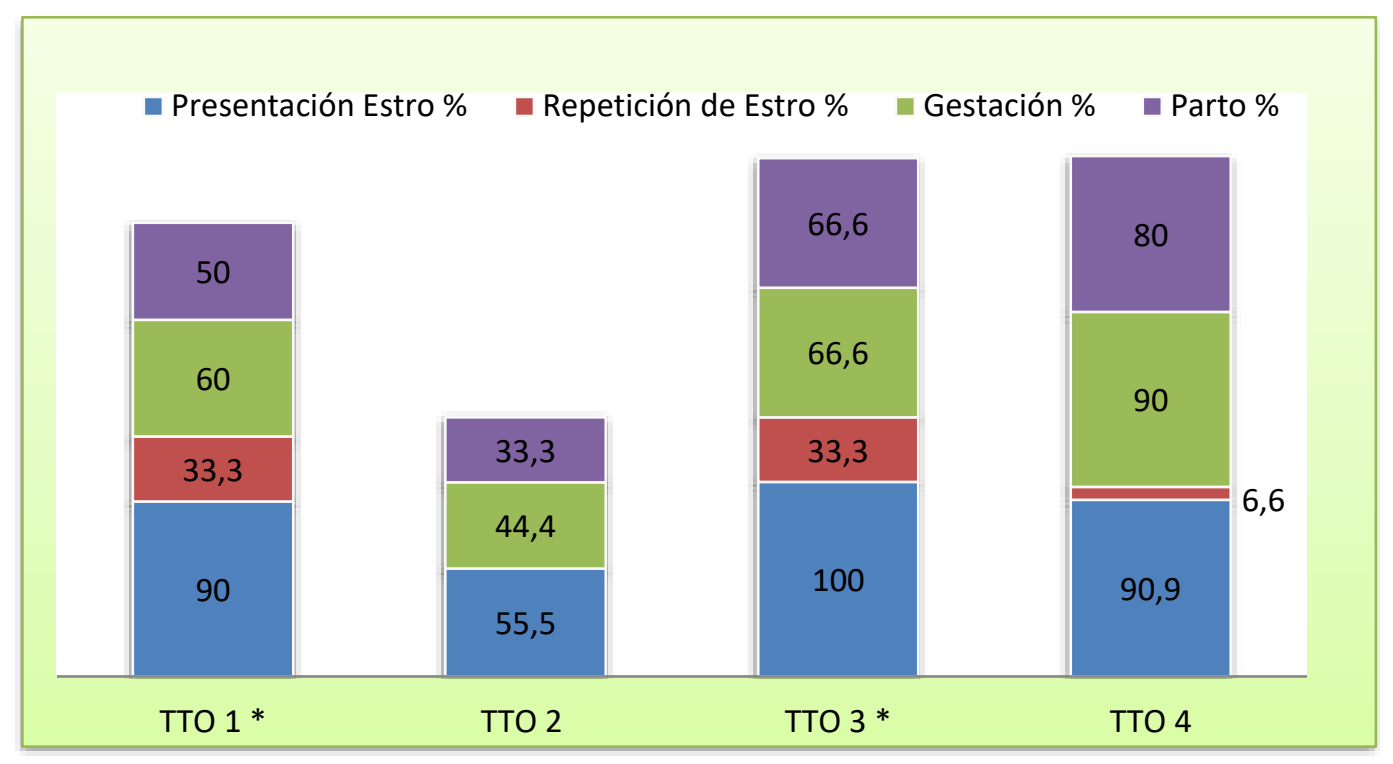

Gráfica 1. Proporciones de variables de desempeño reproductivo en ovejas entre tratamientos. TTO $1^{*}$ y TTO $3^{*}=$ Con flushing.

Con respecto al tiempo de inicio del estro, los tratamientos con CCM (TTO $3^{*}$ y TTO 4) iniciaron la presentación del estro entre la hora 12 y 16 post retiro $\operatorname{CIDR}^{\circledR}$; mientras que las ovejas de CCB (TTO $1^{*}$ y TTO 2), iniciaron presentación del estro entre las 20 y 24 horas pos retiro CIDR $^{\circledR}$. La mayor concentración de ovejas servidas ocurrió 
entre la hora 20 y 32 post retiro $\operatorname{CIDR}^{\circledR}$. Sin embargo, la hora de inicio del estro en este estudio fue menor que el reportado en un estudio realizado en Japón donde se evaluó el inicio del celo con diferentes métodos de sincronización donde la hora en promedio para el inicio de celo con dispositivos $\operatorname{CIDR}^{\circledR}$ fue de 27 horas post retiro, aunque en este estudio no fue considerado la CC (lida et al., 2004).

En cuanto a la repetición del estro los resultados fueron similares entre los grupos TTO $1^{*}(33.3 \%)$ y TTO $3^{*}(33.3 \%)$, mientras entre los tratamientos sin flushing TTO $2(0 \%)$ y TTO $4(6.6 \%)$ fueron diferentes. Estos resultados sugieren que los animales de los $\mathrm{TTO}^{*}$ y $\mathrm{TTO}^{*}$ fisiológicamente ajustaron sus requerimientos nutricionales, los cuales permitieron la presentación de un nuevo estro. A pesar que en un primer servicio no quedaron gestantes, el tratamiento pudo haber influenciado la presentación de un nuevo celo, mientras que en el TTO 2 (CCB sin flushing) el porcentaje de gestación fue del (44.4\%) menor al de presentación del estro (55.5\%) y la repetición de celo fue nula. Algunos autores han relacionado una buena nutrición con una buena calidad, tamaño y cantidad de folículos, incluso en ovejas sobrealimentadas; mientras que en ovejas con mala nutrición el tamaño, cantidad y calidad de folículos fue menor (Fernández et al., 2019; Grazul-bilska et al., 2019).

Por otra parte, el porcentaje de tasa de concepción (TC) fue mayor en el grupo de CCB con flushing; el TTO $1^{*}(60 \%)$ en relación con el TTO2 (44.4\%). En cuanto al grupo de CCM el TTO $3^{*}(66.6 \%)$ obtuvo un valor menor comparándolo con el TTO $4(90.9 \%)$ sin flushing. Estos resultados nos indican que el flushing tuvo un efecto positivo sobre los animales con condición corporal baja, comparado con los animales de condición corporal media (Aguilar et al., 2009; Aké et al., 2013; Camargo, 2018).

La tasa de fertilidad se vio directamente relacionada con la tasa de concepción, igualmente se observó un mayor resultado en el grupo de ovejas con condición corporal baja en el tratamiento con flushing TTO 1* $(50 \%)$, respecto al tratamiento sin flushing TTO 2 (33.3\%). En el grupo de ovejas con condición corporal media se obtuvo igualmente un mayor porcentaje de fertilidad en el tratamiento sin flushing, TTO 4 (81.8\%) respecto al tratamiento con flushing TTO 3 * $(66.6 \%)$. 
Hay que resaltar que sólo en el TTO 3, el 100\% de las ovejas recibió monta, directa, dado que fue el único grupo donde se observó la presentación de estro en la totalidad de los animales, además en este grupo su obtuvo un nacimiento de las crías vivas en su totalidad, a pesar de haber tenido una menor TC y TF en comparación con el TTO 4. Estos resultados podrían indicar una buena fertilidad de las hembras, a la vez la calidad seminal de los machos utilizados, como el tipo de servicio, el cual fue la monta natural. Ha sido reconocido que la fertilidad está ligada a diversos factores como edad de las hembras, la condición corporal, la nutrición y deficiencias de minerales. Ciertos autores han atribuido las altas cargas parasitarias a las pérdidas embrionarias y la disminución de la fertilidad (Fraire et al., 2013; Mantecón et al., 1995).

En dietas suplementadas con ácidos grasos poliinsaturados se obtuvo tasas de concepción del 90\% (Cansino et al., 2009). Por lo anterior en este estudio se lograron resultados similares con la literatura reportada. Sin embrago el TTO 2 alcanzó una TC del (44.4\%) por debajo de lo reportado. Este análisis nos indica que, en ovejas con condición corporal baja, el uso de flushing mejora significativamente la concepción y la fertilidad.

A pesar de que no se logró medir la tasa ovulatoria (TO) en todos los animales de los tratamientos; se midió en un grupo pequeño de animales $(n=4)$ de cada tratamiento para complementar el estudio. La mayor TO fue la de los tratamientos con flushing TTO $1^{*}(2.75)$ y TTO $3^{*}$ (3.25), sin embargo, el TTO 4 (2.5) tuvo una mayor tasa ovulatoria que el TTO $2(1.25)$ el cual tampoco tenía flushing, esto pudo deberse a la baja condición corporal de los animales que componían el TTO 2 respecto al TTO 4. Algunos autores atribuyen el valor de la tasa ovulatoria a la raza (Granados, 2013).

La tasa ovulatoria también está relacionada con la condición corporal de la hembra, la respuesta al flushing en cuanto a la tasa ovulatoria es entre un 15 a $20 \%$ mayor en ovejas con condición corporal de 2.5 a 2.75/5 mientras que en hembras con condición corporal entre 3 y 3.5/5 la respuesta sería de tan sólo un $8 \%$ máximo. Otro factor que influye en el aumento de la tasa ovulatoria hasta en un $6 \%$ es la 
introducción de machos a los lotes de hembras, lo que llamamos comúnmente como el efecto macho (Álvarez et al., 2014).

La prolificidad fue mayor en los grupos donde se instauro el flushing TTO $1^{*}$ (1.3) y TTO $3^{*}$ (1.5). Además, se ha demostrado que el flushing puede mejorar el desempeño reproductivo de los machos antes de la monta (Banchero et al., 2013) y algunos como Aguilar han evaluado el desempeño del flushing en los corderos nacidos de hembras suplementadas con flushing, obteniendo un $22 \%$ más de partos gemelares en hembras suplementadas con un flushing corto vs hembras no suplementadas (Aguilar et al., 2009; Banchero et al., 2013).

Los tratamientos a los cuales se les instauro el flushing TTO $1^{*}$ y TTO $3^{*}$ presentaron mejor desempeño reproductivo. Esta técnica influye positivamente sobre los indicadores reproductivos principalmente en ovejas con baja condición corporal con pocos días post destete, evidenciándose como una estrategia económica y rentable para el rebaño.

\section{CONCLUSIONES}

El flushing mejora la eficiencia de los protocolos de sincronización con CIDR $^{\circledR}$ en hembras ovinas con condición corporal baja, hasta en un $50 \%$. Es necesario corroborar este resultado en diferentes protocolos de sincronización.

Las ovejas con condición corporal media no necesitan la implementación de flushing corto para tener una respuesta positiva a protocolos de sincronización con CIDR, pero es necesario evaluar si esta respuesta fue debido el incremento de la porción de alimento durante el protocolo o no.

El porcentaje de gestación aumentó considerablemente en las ovejas de condición corporal baja cuando son suplementadas con flushing corto antes de la monta, mientras que en las ovejas de condición corporal media a pesar de tener una respuesta mayor en cuanto a la presentación de estros, el porcentaje de gestación no aumenta notablemente en el grupo con flushing. 
La tasa ovulatoria aumentó cuando las ovejas son suplementadas con flushing, a pesar de que la ganancia de peso fue baja.

Las ovejas con condición corporal baja sin flushing, no tienen buena respuesta a la sincronización, el porcentaje de gestación es bajo, la muerte embrionaria es mayor y el porcentaje de partos melliceros es muy bajo.

Se hace necesario en el futuro contar en los nuevos estudios con una mayor muestra de animales, lo cual permita corroborar los resultados encontrados.

El valor de la tasa ovulatoria es sólo una estimación debido al tamaño tan pequeño de la muestra tenida en cuenta; es necesario crear unos valores de referencia por razas, para poder determinar si es alta, baja o promedio, dependiendo la raza.

El flushing es una buena estrategia que se puede aprovechar en la especie ovina de nuestro país, debido a que mejora las variables reproductivas especialmente en ovejas con condición corporal baja o de pocos días post destete, al igual que es económicamente rentable, teniendo en cuenta que algunos autores reportar un mayor peso al nacimiento y desarrollo de las crías de hembras sometidas a flushing.

\section{REFERENCIAS BIBLIOGRÁFICAS}

1. Aguilar, E., Insaurralde, E., Robson, C., Franz, H., Celser, R., Gomez, M. Efecto de la suplementación proteica en ovejas durante el servicio para incrementar la producción de mellizos volver a: Producción ovina en general. Corriedale, Bs. As. Anuario, 0 (1): 4-9. 2009. Disponible En: www.produccion-animal.com.ar

2. Aké, L., Casanova, E., Centurión, F., Aké, V. Efecto de la Condicion corporal sobre la sincronización del estro, fertilidad y prolificidad en ovejas de pelo. 6 (2): 34-38. 2013.

3. Álvarez, E., García, J., Herrmann, F. Tasa ovulatoria de ovejas ideal, con diferentes tratamientos alimenticios previo al servicio (flushing corto), Vol. 53. 2014. Disponible En: https://doi.org/10.1017/CBO9781107415324.004

4. Banchero, G., Quintans, G. "Flushing corto" una herramienta para aumentar el porcentaje de mellizos en ovejas de baja a moderada prolificidad. INIA, N. 14: 1-5. 2008.

5. Banchero, G., Vázquez, A., Quintans, G. Manejo nutricional para aumentar la tasa mellicera en ovinos. 2013.

6. Camargo, D. Suplementación estratégica para mejorar la producción de ovejas en trópico bajo colombiano. 2018. 
7. Cansino, G., Herrera, J., Ake, J. Tasas de concepción, fertilidad y prolificidad en ovejas de pelo alimentadas con dietas enriquecidas con ácidos grasos poliinsaturados. 25 (1): 181-185. 2009.

8. De Azambuja, L., Mori, M., Mizubuti, Y., Das Dores Ferreira Da Silva, L., Do Prado, P., Sales Pereira, E., Fávero, R. Desempenho de cordeiros provenientes de ovelhas de diferentes grupos genéticos e que foram submetidas ao flushing alimentar. Semina: Ciencias Agrarias, 36 (2): 1031-1042. 2015. Disponible En: https://doi.org/10.5433/1679-0359.2015v36n2p1031

9. Felice, M. Condición Corporal de Ovinos. 2013. Disponible En: https://ciencia.lasalle.edu.co/cgi/viewcontent.cgi?article=1359\&context=zootec nia

10. Fernández, A., Sosa, C., Abecia, A., Vázquez, I. Dietary restriction in sheep: Uterine functionality in ewes with different body reserves during early gestation. Theriogenology, 135, 189-197. 2019. Disponible En: https://doi.org/10.1016/j.theriogenology.2019.06.023

11. Fraire, S., Pró Martínez, A., Ramírez, G., Sánchez del Real, C., Gallegos, J., Fraire, S., Gallegos, J. Selenio y vitamina E en la fertilidad de ovejas Pelibuey sincronizadas con progesterona. Universidad y Ciencia, 29 (1): 33-44. 2013. Disponible En: https://doi.org/10.19136/era.a29n1.40

12. Gottardi, P., Souza, A., Barbosa, S., Marques, T., Bezerra, R., Araújo, J., Torreão, C. Efeito do flushing sobre o desempenho reprodutivo de ovelhas Morada Nova e santa inês submetidas à inseminação artificial em tempo fixo. Arquivo Brasileiro de Medicina Veterinaria e Zootecnia, 66 (2): 329-338. 2014. Disponible En: https://doi.org/10.1590/1678-41626103

13. Gozonga, A. Respuesta productiva y características de la canal de ovinos en engorda intensiva suplementados con cromo orgánico y clohidrato de zilpaterol. $2016 . \quad$ Disponibles http://ri.uaemex.mx/bitstream/handle/20.500.11799/58574/TESIS-LAGV0816.pdf? sequence $=1$

14. Granados, V. Evaluación de los efectos de Glicerol por vía oral sobre la Fertilidad de ovejas Rambouillet. Universidad Nacional Autónoma de México. 2013.

15. Grazul-bilska, T., Bass, S., Kaminski, L., Ebel, K., Leke, E., Thammasiri, J., \& Redmer, A. Effects of plane of nutrition and arginine on ovarian follicles in nonpregnant sheep : Cell proliferation, and expression of endothelial nitric oxide and its receptor. Acta Histochemica, 121 (2): 189-197. 2019. Disponible En: https://doi.org/10.1016/j.acthis.2018.12.009

16. lida, K., Kobayashi, N., Kohno, H., Miyamoto, A., Fukui, Y. A comparative study of induction of estrus and ovulation by three different intravaginal devices in ewes during the non-breeding season. Journal of Reproduction and Development, 50 (1): 63-69. 2004. Disponible En: https://doi.org/10.1262/jrd.50.63

17. Mantecón, R., Giráñdez, J., Hervás, L. Requerimientos Nutricionales para Ovinos en Reproducción. 1995.

18. Martínez, E., Carvajal, A., Guarda, P. Preparación para el encaste en las hembras ovinas. 2018. 
19. Martinez, F., McLeod, B., Tattersfield, G., Smaill, B., Quirke, D., Juengel, L. Successful induction of oestrus, ovulation and pregnancy in adult ewes and ewe lambs out of the breeding season using a $\mathrm{GnRH}$ + progesterone oestrus synchronisation protocol. Animal Reproduction Science, 155: 28-35. 2015. Disponible En: https://doi.org/10.1016/i.anireprosci.2015.01.010

20. SAGARPA. Hidalgo; polo de desarrollo pecuario y de producción de carne ovina. 2015. Disponible

En:

https://www.gob.mx/agricultura\%7Chidalgo/articulos/hidalgo-polo-dedesarrollo-pecuario-y-de-produccion-de-carne-ovina-carmen-dorantes

21. SAGARPA. Se coloca la ovinocultura mexicana entre las mejores del mundo. 2020. Recuperado 27 Enero 2020. Disponible En: https://www.gob.mx/agricultura/colima/articulos/se-coloca-la-ovinoculturamexicana-entre-las-mejores-del-mundo-158022?idiom=es

22. Salazar, O. Evaluación de la implementación de Buenas Prácticas Pecuarias en la producción de ovinos y caprinos en la zona metropolitana de los municipios de Bucaramanga y Lebrija. 153. 2015.

23. Sanchez, D., Alberto, P. Efeito da suplementação alimentar pré-acasalamento na prolificidade de ovinos das raças Ideal e Corriedale. Boletim de Indústria Animal, 47 (2): 87-96. 1990. 